\title{
1 Singular spectrum analysis for 2 improving hyperspectral imaging 3 based beef eating quality evaluation
}

4 Tong Qiao ${ }^{a}$, Jinchang Ren ${ }^{b}$, Cameron Craigie ${ }^{c, d}$, Jaime Zabalza ${ }^{e}$, Charlotte Maltin ${ }^{c, f}$, and

$5 \quad$ Stephen Marshall ${ }^{\mathrm{g}}$

${ }^{a, b, e, g}$ Centre for Excellence in Signal and Image Processing (CeSIP), Dept. of Electronic and Electrical Engineering, University of Strathclyde, 204 George Street, Glasgow, G1 1XW, UK.

${ }^{c}$ Quality Meat Scotland, Rural Centre, West Mains, Ingliston Newbridge, EH28 8NZ, UK.

${ }^{d}$ Present address: AgResearch Ruakura, 10 Bisley Road, Hamilton 3240, New Zealand.

${ }^{f}$ Present address: Biomics Ltd, Inverurie, Aberdeenshire, AB51 OLE, UK.

${ }^{a}$ E-mail: t.qiao@strath.ac.uk.

${ }^{b}$ E-mail: jinchang.ren@strath.ac.uk, tel.+44-141-5482384 for corresponding author Dr. Ren.

${ }^{c}$ E-mail: cameron.craigie@agresearch.co.nz.

${ }^{e}$ E-mail:j.zabalza@strath.ac.uk.

${ }^{f}$ E-mail:c.maltin@biomics.co.uk.

${ }^{g}$ E-mail: stephen.marshall@strath.ac.uk.

\section{ABSTRACT}

Detecting beef eating quality in a non-destructive way has been popular in recent years. Among various non-destructive assessing methods, the feasibility of hyperspectral imaging (HSI) system was investigated in this paper. Hyperspectral images of beef samples were collected in an abattoir production line and used for predicting the beef tenderness and $\mathrm{pH}$ value. Support vector machine (SVM) was applied to construct the prediction equation. Before utilizing the original HSI spectral profiles directly, we propose to use singular spectrum analysis (SSA) as a pre-processing approach, where SSA has been proven to be an effective technique for time-series analysis in diverse applications. The results indicate that SSA can remove the instrumental noise of HSI system effectively and therefore improve the prediction performance. 


\section{KEYWORDS}

Hyperspectral imaging, beef quality prediction, singular spectrum analysis, principal component analysis, support vector machine.

\section{Introduction}

As time goes, food quality control has become a significant issue to human beings. Serving as a very important source of nutrition, the quality of muscle foods including meat and fish, influences the re-purchase behavior of consumers (Weeranantanaphan et al., 2011). Considering food quality control requires non-destructive real-time monitoring on the production line, near-infrared spectroscopy (NIRS) was first established as a fast and promising tool for multi-constituent quality analysis of food materials, which has proved its feasibility especially in meat industry (Gowen et al., 2007; Weeranantanaphan et al., 2011). However, with limited spatial information, internal constituent gradients within food products could not be captured by NIRS, leading to discrepancies between predicted and measured composition (Gowen et al., 2007). Therefore, multispectral imaging (MSI) system, combining images at a small number of narrow wavebands, was developed afterwards to overcome the above mentioned drawbacks and has demonstrated its success for detection of meat quality (Dissing et al., 2013; Panagou et al., 2014). Through capturing hundreds of continuous bands at different wavelengths, hyperspectral imaging (HSI), as an updated version of MSI, has received considerable attention in recent years since it can acquire the spatial and spectral information simultaneously. The information contained in the HSI cube can be utilized in many areas, going from traditional applications in agricultural land use analysis with remote sensing (Lee et al., 2010; Prabhakar et al., 2011; Qiao et al., 2014) and military surveillance (Gill et al., 2011; Zhao et al., 2013) to newly emerging platforms for biomedical imaging (Wang et al., 2013) and non-invasive food quality control and analysis (Baiano et al., 2012; Gowen et al., 2009; Kelman et al., 2013; Naganathan et al., 2008; Sun, 
2010). Due to the fact that HSI could collect more information than NIRS and MSI during the same time, there is a growing trend to investigate its ability to predict meat quality in a way that is fast, non-destructive and requires no reagent. In this paper, beef was chosen as a representative of muscle foods since it contributes most to the meat market in EU with $8,000,000$ tonnes annual consumption and similar levels of production (Panagou et al., 2014).

Usually, for both NIRS and HSI, pre-processing of the spectral profile is needed, to eliminate undesired effects and noise produced during the data collection process. Common preprocessing techniques, especially for NIRS, include calculating derivatives, standard normal variate (SNV) and multiplicative scatter correction (MSC) (Rinnan et al., 2009). However, using derivatives of spectra may even enhance the noise and lead to more difficult spectral interpretation. For SNV and MSC, it is required to apply these transformations to all spectra as the corrected spectra would be more accurate if more spectra were involved, which is infeasible in the abattoir. In practice, ideally a prediction model based on HSI will be installed in the abattoir production line, and for every single piece of beef steak, the model should predict the quality assessment result in a real-time manner. Therefore, it is necessary to use a pre-processing technique that can be applied to every single spectrum itself, without considering other spectra. In this paper, we mainly demonstrate that singular spectrum analysis (SSA) can be regarded as an optimal pre-processing step in de-noising HSI beef spectra, where it will not be restricted by the number of HSI samples. With the beef eating quality references available as ground truth, the support vector machine (SVM), which is a state-of-the-art non-linear regression technique, was employed for data regression. Compared to other regression methods, SVM does not ask for a large amount of training samples to construct the calibration equation. Additionally, it is not affected by sample outliers either (Burges, 1999). 

The remaining sections of this paper are organized as follows. In Section 2, sample preparation and collection are presented. Besides, algorithms employed in the experiments will be explained as well. Experimental results and conclusion are given in Section 3 and 4 respectively.

\section{Materials and methods}

\subsection{HSI system}

A push-broom HSI system (Gilden photonics) with wavelength ranging from 283 to $863 \mathrm{~nm}$ at a spectral resolution of about $2.5 \mathrm{~nm}$ was used to collect beef data samples. Fig. 1 shows a schematic diagram of the imaging system, which consists of a charge-coupled device (CCD) camera, a spectrograph with lens that utilizes a wavelength dispersive system to acquire all wavelengths of a single spatial line simultaneously, a tungsten halogen lamp, a sliding track and a black tray for the beef. With the targeted object sliding through the imaging system, a three dimensional HSI cube can be formed. Before the image collection starts, a spectral calibration procedure has to be done at two extreme illuminating conditions, using a white tile that reflects almost $100 \%$ of the radiation at all working wavelengths and also the lens cap to get a dark image. These steps make sure that the sample reflectance can be separated from the system response (Naganathan et al., 2008). Eq.(1) shows the calibration calculation,

$$
R=\frac{I-B}{I_{W}-B}
$$

where $I, I_{w}$ and $B$ stand for intensities of the raw image and white/dark reference images, respectively. 


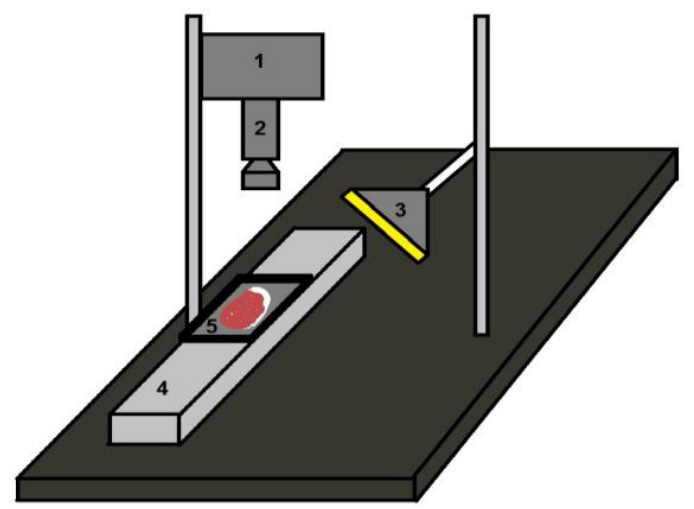

Fig. 1. Schematic diagram of a visible HSI system: components 1-5 refer to the CCD camera, spectrograph and lens, halogen lamp, sliding track and scanning tray, respectively.

\subsection{Sample preparation and HSI data collection}

Over 200 carcasses (M. longissimus muscle), which are aged for 48 hours, were randomly selected in an abattoir production line during two consecutive days, irrespective of gender, conformation, fatness, weight or maturity. For each carcass, a piece of steak with thickness of $25 \mathrm{~mm}$ was recovered from the $11^{\text {th }}$ rib position of the strip loin.

Allowing for two minutes of blooming, hyperspectral images were collected. After imaging, each steak was divided into lateral and dorsal halves, labelled and vacuum packaged. The lateral halves were further aged for 5 days and the dorsal halves were aged for 12 days at -1 ㅇ $\mathrm{C}$ before freezing. A temperature data logger was packed with each batch in order to verify the temperature during the aging process. Thus, these steaks had a total aging time of 7 days and 14 days before quality parameter measurements.

\subsection{Sample quality reference measurements}

Tenderness, juiciness and flavor of beef are considered as the most important attributes that influence the repurchase behavior of consumers (Shackelford et al., 2001). In our experiments, slice shear force (SSF) was measured as the tenderness reference, while the ultimate $\mathrm{pH}$ is found to have a strong relationship with juiciness and flavor of beef steaks. On the day before tenderness and $\mathrm{pH}$ tests, steaks were thawed overnight at ambient 
temperature and ultimate $\mathrm{pH}$ values were first measured using a calibrated Hanna meat $\mathrm{pH}$ meter ( $\mathrm{HI}$ 99163) without the knife blade attached. Two measurements were taken at different locations and averaged to give the final result. For offline SSF measurement, steaks were cooked on a clam-shell grill until the center temperature reached $71 \stackrel{\circ}{\circ}$ using a stainless steel temperature probe. Samples were sheared perpendicular to the muscle fiber axis with a Tenderscot tenderometer (Pentland Precision Engineeris, Loanhead, Midlothian), and the highest force during the shear process was picked up as the SSF. In summary, there are four beef quality attributes in total for each steak that need to be predicted, which are SSF7, SSF14, $\mathrm{pH} 7$ and $\mathrm{pH} 14$.

\subsection{Singular spectrum analysis}

As a relatively new technique, SSA is commonly used for time series analysis and forecasting. Based on the singular value decomposition (SVD), it is able to decompose the original time series into a few components, including the 'clean' series, oscillations and noise (Zabalza et al., 2014b). The algorithm of SSA is briefly introduced as follows.

\section{The first step in the SSA algorithm is to transform the investigated series into the trajectory} matrix. Assume we have a one dimensional series vector with length $N$ as $\mathbf{X}=\left(x_{1}, \cdots, x_{N}\right)$. Given a window length $L(1<L<N)$, the initial series can be mapped into $K$ lagged vectors, $\mathbf{X}_{i}=\left(x_{i}, \cdots, x_{i+L-1}\right)^{\top}$ for $i=1, \cdots, K$, where $K=N-L+1$. Then, the trajectory matrix is formed as Eq.(2). One thing worth of noting is that the matrix $\mathbf{T}$ is a Hankel matrix with size of $L \times K$, where $\mathbf{T}$ has equal elements $x_{i j}$ on the anti-diagonals where $i+j=$ const,

$$
\mathbf{T}=\left(\begin{array}{llll}
\mathbf{X}_{1} & \mathbf{X}_{2} & \cdots & \mathbf{X}_{K}
\end{array}\right)=\left(\begin{array}{cccc}
x_{1} & x_{2} & \cdots & x_{K} \\
x_{2} & x_{3} & \cdots & x_{K+1} \\
\vdots & \vdots & \ddots & \vdots \\
x_{L} & x_{L+1} & \cdots & x_{N}
\end{array}\right) .
$$


139 The next step is to perform the SVD of the trajectory matrix $\mathbf{T}$. First, the eigenvalues of $\mathbf{T T}^{\top}$

140 are calculated and arranged in the decreasing order, written as $\left(\lambda_{1} \geq \cdots \lambda_{L} \geq 0\right)$. The 141 corresponding eigenvectors are denoted as $\left(U_{1}, \cdots, U_{L}\right)$. Then the result of SVD is shown in 142 Eq.(3),

$$
\mathbf{T}=\mathbf{T}_{1}+\cdots+\mathbf{T}_{d}
$$

144 where $d$ is the rank of $\mathbf{T}, \mathbf{T}_{i}=\sqrt{\lambda_{i}} \mathbf{U}_{i} \mathbf{V}_{i}^{\top}(i=1, \cdots, d)$ are called elementary matrix with rank 1451 , and $\mathbf{V}_{i}=\mathbf{T}^{\mathbf{T}} \mathbf{U}_{i} / \sqrt{\lambda_{i}}$ are often called principal components of the matrix $\mathbf{T}$. In general, the contribution of $\mathbf{T}_{i}$ to the trajectory matrix $\mathbf{T}$ depends on the ratio of each eigenvalue and the sum of these eigenvalues, shown in Eq.(4),

$$
\eta_{i}=\lambda_{i} / \sum_{i=1}^{d} \lambda_{i}
$$

149 The following step of SSA is called grouping, where the set of indices $\{1, \cdots, d\}$ is divided into $150 m$ disjointed subsets $I_{1}, \cdots, I_{m}$. Assume $I=\left\{i_{1}, \cdots, i_{p}\right\}$, then the trajectory matrix corresponding to the group $I$ is defined as $\mathbf{T}_{I}=\mathbf{T}_{i_{1}}+\cdots+\mathbf{T}_{i_{p}}$. Similarly, the resultant trajectory matrices can be calculated for every group $I=I_{1}, \ldots, I_{m}$ and Eq.(3) is expanded as Eq.(5), The last step is the diagonal averaging which first hankelizes the grouped matrices $\mathbf{T}_{1}$ and 155 then transforms them into a new series with length $N$. Let $Y_{1}=\left(y_{1}, \cdots, y_{N}\right)$ be the transformed one dimensional series of $\mathbf{T}_{l_{1}}$, elements in $\mathbf{Y}_{1}$ can be represented as Eq.(6), 


$$
y_{k}=\left\{\begin{array}{cc}
\frac{1}{k} \sum_{j=1}^{k} y_{j, k-j+1}^{*}, & \text { for } \quad 1 \leq k<L^{*} \\
\frac{1}{L^{*}} \sum_{j=1}^{L^{*}} y_{j, k-j+1}^{*}, & \text { for } \quad L^{*} \leq k \leq K^{*} \\
\frac{1}{N-k+1} \sum_{j=k-K^{*}+1}^{N-K^{*}+1} y_{j, k-j+1}^{*}, & \text { for } \quad K^{*}<k \leq N,
\end{array}\right.
$$

158

where $L^{*}=\min (L, K), K^{*}=\max (L, K), y_{j, k-j+1}^{*}=y_{j, k-j+1}$ if $L<K$ and $y_{j, k-j+1}^{*}=y_{k-j+1, j}$ if $L \geq K$. Hence, the initial series $\mathbf{X}=\left(x_{1}, \cdots, x_{N}\right)$ can be decomposed into $m$ series:

$$
\mathbf{X}=\mathbf{Y}_{1}+\cdots+\mathbf{Y}_{m} .
$$

In SSA decomposition with $m$ reconstructed series, the first reconstructed series $\left(\mathbf{Y}_{1}\right)$ is regarded as the most important one. Hence the rest are discarded as noise.

\subsection{HSI data pre-processing}

The hyperspectral image data were then imported to MATLAB 2013 (MathWorks) for further processing and analysis. Fig. 2(a) shows a sample image at one wavelength after reflectance calibration. In order to improve the regression performance and processing speed, a regionof-interest (ROI) with size of $100 \times 200$ was selected from the lean part on each hyperspectral image, illustrated in Fig. 2(b). Even though most of fat area was discarded, there were still a few pixels that had different spectral features from pure lean pixels, i.e. dead pixels. In this context, an iterative pixel removal process (Burger and Geladi, 2006) was applied to the ROI to exclude these dead pixels. For all spectra in the ROI, the Euclidean distances to the median spectrum and the standard deviation of these distances were calculated. Then those pixels with distances higher than five standard deviations were removed from the ROI. It is assumed that in our experiments, 100 times iterations should be enough for removing dead pixels. In addition, the first 50 bands were removed due to the visual spatial noise presented in the images. After these cleaning steps, it is assumed that all pixels in the ROI are contributed by the lean part and finally a new median spectrum in Fig. 

median spectrum, the reflectance at each wavelength does not necessarily come from the same location in the ROI. As a result, noise could also possibly be produced during this process. As mentioned previously, we propose to use SSA for removing various noises from HSI median spectra and the result is shown by the dotted line in Fig. 2(c).

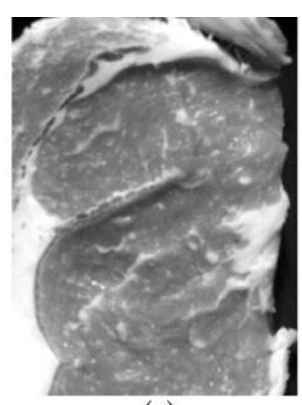

(a)

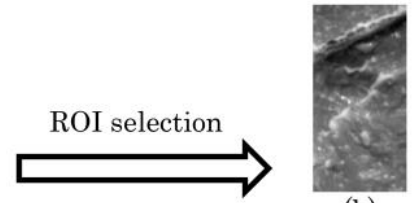

(b)

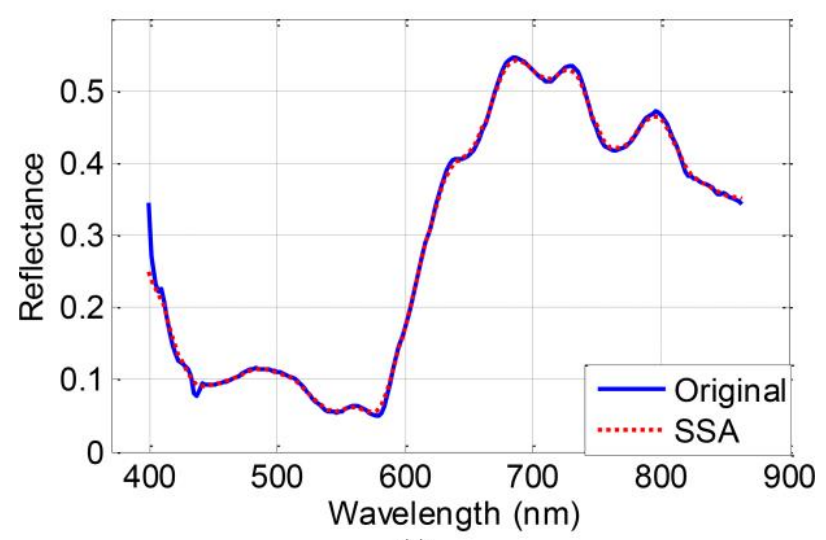

(c)

Fig. 2 HSI spectral feature extraction. (a) A band in a sample hyperspectral image, (b) ROI in the sample image, and (c) original median spectrum and SSA treated median spectrum of the ROI.

There are two important parameters that affect the performance of SSA, which are the window length $L$ and the eigenvalue grouping. The window length determines the number of decomposed series after SSA, while the eigenvalue grouping defines how many

189 decomposed series are used for reconstruction in order to remove noise. In our experiments, 190 we only used the first decomposed series for spectrum reconstruction as previously 191 discussed and the only parameter to be adjusted is the window length $L$. In Fig. 2(c), the 
window length is set as 5 . Even though a subtle difference can be noticed in the plot, it is demonstrated that SSA did improve the prediction performance as shown below.

After effective feature extraction by SSA, the reflectance spectra $(R)$ were usually transformed into absorbance $(1 / R)$ by logarithm transformation, in order to linearize the relationship between the concentration of an absorbing compound and the absorption spectrum (Rust et al., 2008).

\subsection{Regression analysis model}

Unlike other researchers who usually use partial least squares regression (PLSR) to build the regression model, we have employed SVM instead. The ability of SVM has been exploited in many applications associated with $\mathrm{HSI}$, which is proved to be an outstanding machine learning model. However, a major problem of SVM is its curse of dimensionality. As a result, principal component analysis (PCA), as an effective feature extraction technique for hyperspectral data (Gowen et al., 2008; Ren et al., 2014; Zabalza et al., 2014a; Zabalza et al., 2014c), was introduced to reduce the dimensionality of our beef hyperspectral images.

PCA is found to be able to project the high-dimensionality data to a new space, where differentiability is higher in a subset containing first few transformed features than other subsets (Sun, 2010). Therefore, only a small amount of features would explain the highdimensionality data and the rest of features can be discarded.

Assume $\mathbf{x}_{k}(k=1,2, \cdots, n)$ is the reflectance spectrum of each sample with $m$ dimensions, then the whole dataset can be represented by an $n \times m$ matrix, shown as Eq.(8):

$$
\mathbf{X}=\left(\begin{array}{c}
\mathbf{x}_{1} \\
\mathbf{x}_{2} \\
\vdots \\
\mathbf{x}_{n}
\end{array}\right) \text {. }
$$


The first step of PCA is to find the covariance matrix of the whole data, as given in Eq.(9),

$$
\mathbf{S}_{\mathbf{x}}=\frac{1}{n-1}(\mathbf{X}-\boldsymbol{\mu})^{\top}(\mathbf{X}-\boldsymbol{\mu})
$$

where $\boldsymbol{\mu}$ is the mean spectrum of the whole dataset. The projection $\mathbf{W}_{\text {opt }}$ is chosen to maximize the determinant of the total covariance matrix of the projected samples, which is

$$
\mathbf{W}_{\text {opt }}=\operatorname{argmax}\left(\mathbf{W}^{\top} \mathbf{S}_{\mathbf{x}} \mathbf{W}\right)=\left(\begin{array}{llll}
\mathbf{w}_{1} & \mathbf{w}_{2} & \cdots & \mathbf{w}_{L}
\end{array}\right),
$$

where $\mathbf{w}_{i}(i=1,2, \ldots, L)$ is the set of $L$ dimensional eigenvectors of $\mathbf{S}_{\mathbf{x}}$ corresponding to $L$ largest eigenvalues. Thus, the dimensionality is reduced from $m$ to $L$.

With a lower dimension $L, S V M$ is applied to construct the prediction model. For classification and regression problems, SVM maps the training dataset $\mathbf{x}$ to a high dimensional feature space by using a non-linear kernel function, shown below:

$$
K\left(\mathbf{x}_{i}, \mathbf{x}_{j}\right)=\Phi\left(\mathbf{x}_{i}\right) \Phi\left(\mathbf{x}_{j}\right),
$$

224

where $\Phi$ is the mapping function. Then it is able to classify the data by a maximal margin hyperplane. The radial basis function (RBF) kernel was selected in this paper, given in Eq.(12):

$$
K\left(\mathbf{x}_{i}, \mathbf{x}_{j}\right)=\exp \left(-\gamma\left\|\mathbf{x}_{i}-\mathbf{x}_{j}\right\|^{2}\right)
$$

Optimized parameters were grid-searched using four-fold cross-validation in order to avoid model over-fitting.

\section{Results and discussion}

To test the ability of HSI for evaluating unknown beef quality, data has to be split into the calibration set and the validation set, where models would be learnt from the calibration set and tested on the validation set. By sorting each quality trait (SSF7, SSF14, $\mathrm{pH} 7$ and $\mathrm{pH} 14$ ) in 
ascending order respectively and selecting every fourth sample into the validation set with

234 the interleaving three samples being allocated to the calibration set (Williams, 2001), it

235 ensures that the validation set is similar to the calibration set, considering the mean value,

236 the range and the standard deviation (SD) of each analyzed quality attribute, where the

237 statistics are shown in Table 1.

Table 1. Summary statistics of studied beef quality attributes.

\begin{tabular}{|c|c|c|c|c|c|c|c|c|c|c|}
\hline \multirow[t]{2}{*}{ Trait } & \multicolumn{5}{|c|}{ Calibration set } & \multicolumn{5}{|c|}{ Validation set } \\
\hline & $\mathrm{n}$ & Min & Max & Mean & SD & $\mathrm{n}$ & Min & Max & Mean & SD \\
\hline SSF7 & 159 & 46.97 & 299.54 & 131.46 & 48.18 & 52 & 69.41 & 285.62 & 130.73 & 45.69 \\
\hline SSF14 & 159 & 63.35 & 291.56 & 132.23 & 42.91 & 52 & 73.61 & 239.82 & 131.32 & 39.91 \\
\hline $\mathrm{pH} 7$ & 154 & 5.44 & 6.37 & 5.63 & 0.13 & 51 & 5.46 & 6.34 & 5.63 & 0.14 \\
\hline $\mathrm{pH} 14$ & 154 & 5.46 & 6.46 & 5.69 & 0.14 & 51 & 5.48 & 6.41 & 5.69 & 0.14 \\
\hline
\end{tabular}

239

240 The prediction performance was evaluated quantitatively by the coefficient of

241 determination $\left(R^{2}\right)$ and the ratio of performance deviation $(R P D)$. Equations for these

242 metrics are given below:

$$
\begin{gathered}
R^{2}=1-\frac{\sum_{i=1}^{n}\left(y_{i}-f_{i}\right)^{2}}{\sum_{i=1}^{n}\left(y_{i}-\bar{y}\right)^{2}}, \\
R P D=\frac{S D}{R M S E P}=\frac{S D}{\sqrt{\frac{\sum_{i=1}^{n}\left(f_{i}-y_{i}\right)^{2}}{n}}},
\end{gathered}
$$

245 where $y_{i}$ is the original quality trait value, $f_{i}$ is the predicted trait value, $\bar{y}$ is the mean of 246 original trait values and $n$ is the total number of samples in the validation set. The 247 coefficient of determination varies within 0 to 1 , where 0 represents a poor correlation 
between the predicted trait values and the reference trait values while 1 means a high degree of correlation.

By trial and error, the number of principal components was set as 30 . In that case,

251 dimensionality has been reduced dramatically while enough features have been preserved.

252 Results comparing the performance of the original spectra and SSA treated spectra for the

253 validation set are shown in Table 2 . The window length $L$ was tuned to achieve the best

254 result. As highlighted in bold, except for limited improvement on SSF14, SSA could always

255 stand out prominently, which demonstrates that it is an excellent feature extraction and de-

256 noising pre-processing procedure for HSI spectral profiles. Just like the time series, in this

257 paper one-dimensional (1D) SSA was applied in HSI spectral domain. Future work involves

258 combining two-dimensional (2D) SSA on the spatial domain, which has been proved to be

259 even more effective on remote sensing HSI based classification problems than 1D SSA

260 (Zabalza et al., 2015).

261

262

\begin{tabular}{|c|c|c|c|c|c|}
\hline \multirow[t]{2}{*}{ Trait } & \multicolumn{2}{|c|}{ Original spectra } & \multicolumn{3}{|c|}{ SSA treated spectra } \\
\hline & $\mathrm{R}^{2}$ & RPD & $\mathrm{L}$ & $\mathrm{R}^{2}$ & RPD \\
\hline SSF7 & 0.1938 & 1.1019 & 2 & 0.3288 & 1.2082 \\
\hline SSF14 & 0.1001 & 1.0264 & 2 & 0.1104 & 1.0249 \\
\hline $\mathrm{pH} 7$ & 0.4227 & 1.2490 & 3 & 0.4511 & 1.2822 \\
\hline $\mathrm{pH} 14$ & 0.2785 & 1.1234 & 7 & 0.3419 & 1.2090 \\
\hline
\end{tabular}

264 If we adjust the number of principal components and the window length of SSA by

265 conducting a grid search, the regression performance with the validation set can be even

266 further improved. Chosen parameters and results for SSA treated HSI spectra are shown in

267 Table 3. 


\begin{tabular}{lllll}
\hline Trait & No. of PCS & $\mathrm{L}$ & $\mathrm{R}^{2}$ & $\mathrm{RPD}$ \\
\hline SSF7 & 30 & 2 & 0.3288 & 1.2082 \\
$\mathrm{SSF14}$ & 20 & 6 & 0.2033 & 1.1048 \\
$\mathrm{pH7}$ & 45 & 2 & 0.5838 & 1.4320 \\
$\mathrm{pH} 14$ & 45 & 3 & 0.4863 & 1.3615 \\
\hline
\end{tabular}

As can be seen, for predicting ultimate $\mathrm{pH}$ values, both $R^{2}$ and $R P D$ indicate that $\mathrm{HSI}$ could be

271 a promising tool for the beef industry. However, the results for predicting SSF are not very

272 satisfying, which might be due to the fact that SSF is not a standard quantitative metric of

273 meat tenderness, regardless inconsistent measurements within the ground truth. Similar

274 results have been achieved by other researchers as well. Prieto et al. (Prieto et al., 2009)

275 have predicted SSF using NIRS, giving $R^{2}$ of $0.31(R P D=1.25)$ and $0.23(R P D=1.14)$ for SSF3

276 and SSF14 respectively. But, it should be noted that comparisons between studies are not

277 reliable due to different samples used. Nevertheless, we cannot deny the potential of HSI in

278 providing additional information that could help to improve prediction of meat quality

279 attributes.

\section{Conclusion}

281 In conclusion, a relatively new time series analysis method named SSA is proposed to pre-

282 process HSI spectra. By decomposing original spectra and reconstructing the spectra using 283 the most significant components, SSA demonstrates its ability in removing noise and 284 improving the prediction accuracy for HSI based beef eating quality evaluation. Although in 285 this paper SSA was only applied on beef hyperspectral images, it is not limited in this field.

286 We believe that it can be extended to other HSI based applications, including data prediction 287 in both classification and regression problems. 


\section{Acknowledgement}

The current research is funded by Quality Meat Scotland (QMS) and University of Strathclyde. Authors would like to thank editors and anonymous reviewers for helping improving the quality of this paper.

\section{References}

Baiano, A., Terracone, C., Peri, G., Romaniello, R., 2012. Application of hyperspectral imaging for prediction of physico-chemical and sensory characteristics of table grapes. Comput. Electron. Agric. 87, 142-151.

Burger, J., Geladi, P., 2006. Hyperspectral NIR image regression part II: Dataset preprocessing diagnostics. J. Chemometr. 20 (3-4), 106-119.

Burges, C., 1999. Geometry and invariance in kernel based methods. MIT Press, Cambridge, MA.

Dissing, B., Papadopoulou, O., Tassou, C., Ersboll, B., Carstensen, J., Panagou, E., Nychas, G., 2013. Using multispectral imaging for spoilage detection of pork meat. Food Bioprocess Tech. $6(9), 2268-2279$.

Gill, K., Ren, J., Marshall, S., Karthick, S., Gilchrist, J., 2011. Quality-assured fingerprint image enhancement and extraction using hyperspectral imaging. In: Proceedings of the 4th International Conference on Imaging for Crime Detection and Prevention 2011 (ICDP 2011), London.

Gowen, A., O'Donnell, C., Cullen, P., Downey, G., Frias, J., 2007. Hyperspectral imaging - an emerging process analytical tool for food quality and safety control. Trends Food Sci. Tech. 18, 590-598.

Gowen, A., O’Donnell, C., Taghizadeh, M., Cullen, P., Frias, J., Downey, G., 2008. Hyperspectral imaging combined with principal component analysis for bruise damage detection on white mushrooms (Agaricus bisporus). J. Chemometr. 22 (3-4), 259-267.

Gowen, A., Taghizadeh, M., O'Donnell, C., 2009. Identification of mushrooms subjected to freeze damage using hyperspectral imaging. J. Food Eng. 93 (1), 7-12.

Kelman, T., Ren, J., Marshall, S., 2013. Effective classification of Chinese tea samples in hyperspctral imaging. Artif. Intell. Res. 2, 87-96.

Lee, W.S., Alchanatis, V., Yang, C., Hirafuji, M., Moshou, D., Li, C., 2010. Sensing technologies for precision specialty crop production. Comput. Electron. Agric. 74 (1), 2-33. 
Naganathan, G.K., Grimes, L.M., Subbiah, J., Calkins, C.R., Samal, A., Meyer, G.E., 2008. Visible/near-infrared hyperspectral imaging for beef tenderness prediction. Comput. Electron. Agric. 64, 225-233.

Panagou, E., Papadopoulou, O., Carstensen, J., Nychas, G.J., 2014. Potential of multispectral imaging technology for rapid and non-destructive determination of the microbiological quality of beef filets during aerobic storage. Int. J. Food Microbiol. 174, 1-11.

Prabhakar, M., Prasad, Y.G., Thirupathi, M., Sreedevi, G., Dharajothi, B., Venkateswarlu, B., 2011. Use of ground based hyperspectral remote sensing for detection of stress in cotton caused by leafhopper (Hemiptera: Cicadellidae). Comput. Electron. Agric. 79 (2), 189-198.

Prieto, N., Ross, D., Navajas, E., Nute, G., Richardson, R., Hyslop, J., Simm, G., Roehe, R., 2009. On-line application of visible and near infrared reflectance spectroscopy to predict chemical-physical and sensory characteristics of beef quality. Meat Sci. 83 (1), 96-103

Qiao, T., Ren, J., Sun, M., Zheng, J., Marshall, S., 2014. Effective compression of hyperspectral imagery using an improved 3D DCT approach for land-cover analysis in resensing applications. Int. J. Remote Sens. 35 (20), 7316-7337.

Ren, J., Zabalza, J., Marshall, S., Zheng, J., 2014. Effective feature extraction and data reduction in remote sensing using hyperspectral imaging. IEEE Signal Process. Mag. 31 (4), 149-154.

Rinnan, A., van den Berg, F., Engelsen, S., 2009. Review of the most common pre-processing techniques for near-infrared spectra. TRAC-Trend. Anal. Chem. 28 (10), 1201-1222.

Rust, S., Price, D., Subbiah, J., Kranzler, G., Hilton, G., Vanoverbeke, D., Morgan, J., 2008. Predicting beef tenderness using near-infrared spectroscopy. J. Anim. Sci. 86 (1), 211-219

Shackelford, S., Wheeler, T., Meade, M., Reagan, J., Byrnes, B., Koohmaraie, M., 2001. Consumer impressions of Tender Select beef. J. Anim. Sci. 79 (10), 2605-2614.

Sun, D.W., 2010. Hyperspectral imaging for food quality analysis and control. Elsevier.

Wang, T., Li, Q., Li, X., Zhao, S., Lu, Y., Huang, G., 2013. Use of hyperspectral imaging for label-free decoding and detection of biomarkers. Opt. Lett. 38 (9), 1524-1526.

Weeranantanaphan, J., Downey, G., Allen, P., Sun, D.W., 2011. A review of near infrared spectroscopy in muscle food analysis: 2005-2010. J. Near Infrared Spectrosc. 19 (2), 61-104.

Williams, P., 2001. Near-infrared technology in the agricultural and food industries. American Association of Cereal Chemists, St. Paul, Minnesota, USA.

Zabalza, J., Ren, J., Ren, J., Liu, Z., Marshall, S., 2014a. Structured covariance principal component analysis for real-time onsite feature extraction and dimensionality reduction in hyperspectral imaging. Appl. Opt. 53 (20), 4440-4449 
353 Zabalza, J., Ren, J., Wang, Z., Marshall, S., Wang, J., 2014b. Singular spectrum analysis for

354 effective feature extraction in hyperspectral imaging. IEEE Geosci. Remote Sens. Lett. 11 (11), 355 1886-1890.

356 Zabalza, J., Ren, J., Yang, M., Zhang, Y., Wang, J., Marshall, S., Han, J., 2014c. Novel folded357 PCA for improved feature extraction and data reduction with hyperspectral imaging and SAR 358 in remote sensing. ISPRS J. Photogramm. Remote Sens. 93 (7), 112-122.

359 Zabalza, J., Ren, J., Zheng, J., Han, J., Zhao, H., Li, S., Marshall, S., 2015. Novel two360 dimensional singular spectrum analysis for effective feature extraction and data 361 classification in hyperspectral imaging. IEEE Trans. Geosci. Remote Sens. 53 (8), 4418-4433.

362 Zhao, C., Li, X., Ren, J., Marshall, S., 2013. Improved sparse representation using adaptive 363 spatial support for effective target detection in hyperspectral imagery. Int. J. Remote Sens. $36434(24), 8669-8684$. 